\title{
THE EFFECT OF ELECTRON BEAMS ON THE HYDROGEN EMISSION IN IMPULSIVE FLARES ON THE SUN AND STARS
}

\author{
V.V. ZHARKOVA AND V.A.KOBYLINSKY \\ Physics Department, Kiev University, Glushkova 6, Kiev 252022, Ukraine
}

\section{INTRODUCTION}

Flares are very frequent phenomena on the sun and on other stars and are actively investigated with precise observational techniques. These studies are particularly stimulated by recent X-ray and UV observations on spacecraft (Skylab, SMM). From all these observations the flares on the sun and stars seem to be of the same physical nature but of different energetic power. A number of investigations devoted to comparison between the solar and stellar flares led to the conclusion that they have the similar mechanisms for the energy release and transport and for their manifestations in different ranges of electromagnetic radiation.

The hydrogen emission is easy to observe in these events, and thus it is convenient for diagnostic analysis of physical conditions in the flares. However, the impulsive events were considered to be observed in X-rays so only. Zharkova \& Kobylinsky $(1992,1993)$ have recently shown that the hydrogen continuous radiation of the solar flares is sensitive to the electron beam parameters. We also develop an approach to detection of the flares of non-thermal or thermal origin by observing the hydrogen Balmer and Pashen continuum emissions with high temporal resolution.

\section{FORMATION OF THE HYDROGEN EMISSION IN IMPULSIVE FLARES}

The hydrogen emission in elementary flare bursts is originated during a very short period of the electron beam injection. We consider the first few seconds of the injection, during which the hydrogen emissions in UV and in the optical spectrum are appear to be correlate with the hard X-rays. The state of the flaring plasma is described simultaneously by gas-dynamic equations and radiative transfer, statistical equilibrium and particle conservation equations. As a first approximation we use a solution of the gas-dynamic problem obtained by Somov et al. (1981) for an appropriate set of the beam parameters $F_{0}$ and $\gamma$. Then we calculate the hydrogen line and continuum intensities by solving the full nonLTE problem. The technique is described in details by Zharkova \& Kobylinsky (1991).

We consider the electron beam effects on the flaring atmosphere for the initial energy flux of electrons with energy above $20 \mathrm{keV}$ on a top boundary $F_{0}=10^{9}$ and $10^{11} \mathrm{erg} \mathrm{cm}^{-2} \mathrm{~s}^{-1}$ and for the X-ray power index $\gamma=3$ and 5 . These values are in agreement with the solar observations and the return current stability limit of an electron beam (Aboudarham \& Henoux, 1986; Zharkova 


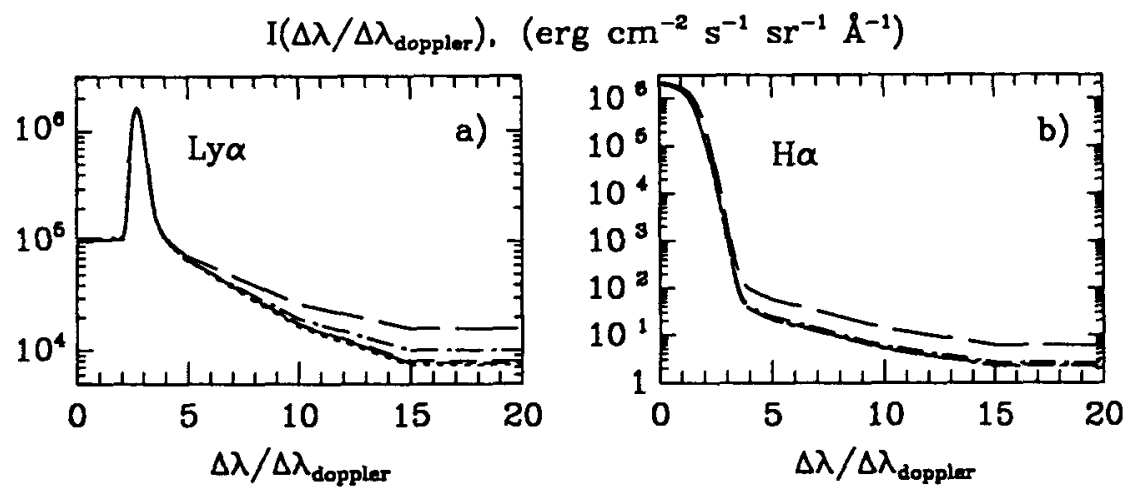

FIGURE I The hydrogen Ly $\alpha$ and $\mathrm{H} \alpha$ line intensities computed for the gas-dynamic flare models at time $2.5 \mathrm{~s}$ : a pure thermal model (dotted curves); models with energetic electrons: $\gamma=3, F_{0}=10^{9} \mathrm{erg} \mathrm{cm}^{-2} \mathrm{~s}^{-1}$ (short dashes); $\gamma=3, F_{0}=10^{11}$ (long dashes); $\gamma=5, F_{0}=10^{9}$ (dot-short-dashed lines); $\gamma=5, F_{0}=10^{11}$ (dot-long-dashed lines)
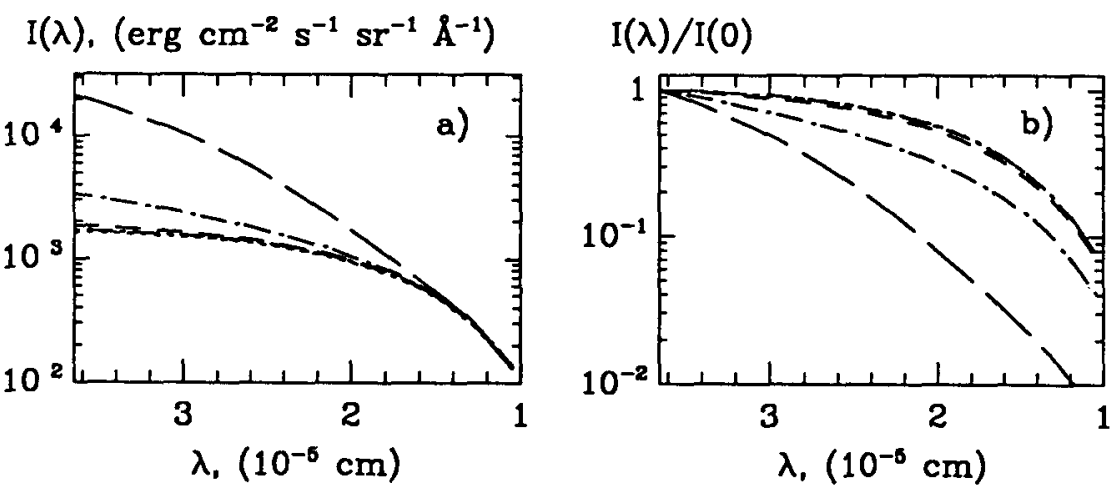

FIGURE II The intensity distributions $(a)$ and slopes $(b)$ of the Bacontinuum at $2.5 \mathrm{~s}$ for the same flare models as in Figure I.

\& Kobylinsky, 1992). For other stars the higher values of $F_{0}=10^{12}-10^{13} \mathrm{erg}$ $\mathrm{cm}^{-2} \mathrm{~s}^{-1}$ should be used. In Figure I we illustrate the hydrogen Ly $\alpha$ and $\mathrm{H} \alpha$ lines intensities and in Figure II the intensity distribution and slope in the head of the $\mathrm{Ba}$ continuum computed for the gas-dynamic models at $2.5 \mathrm{~s}$ after the beginning of electron beam injection. Immediately after the beam injection the nonthermal effects are confined in the high chromosphere. After the gas-dynamic response of the chromosphere $(t>4 \mathrm{~s})$ the influences of the energetic electrons on the line profiles and continuum intensity distributions and their slopes depend strongly on physical conditions in the flares and on beam parameters.

Hydrogen Ly $\alpha$ and $\operatorname{Ly} \beta$ line profiles vary rather similarly. During the first second the line core intensities decrease for low $F_{0}\left(10^{9}\right)$ and increase for higher $F_{0}\left(10^{11}\right)$ as compared with the pure thermal model. In contrast the wing intensities depend strongly on the spectral index $\gamma$ and are less sensitive to the initial flux $F_{0}$, increasing for smaller $\gamma$ and higher $F_{0}$. At $2.5 \mathrm{~s}$ after the injection 
the Ly-line profiles show a significant self-absorption in higher intensities at smaller $\gamma$. After $4 \mathrm{~s}$ the Ly-line profiles begin to return to their form without the self-absorption. The $\mathrm{H} \alpha$-line profiles (see Figure I) become broader and their central intensities become lower with $F_{0}$ increasing and $\gamma$ decreasing. The most significant effects occur in the line wings, where for the large fluxes $\left(10^{11}\right.$ erg $\mathrm{cm}^{-2} \mathrm{~s}^{-1}$ ) the intensity increases by factor 2 as compared with the thermal model. We conclude that the beam electrons affect the hydrogen lines mainly in their wings, and that rather precise observations are needed to detect the effects.

The hydrogen continuum intensities are much more sensitive to non-thermal impacts. The Ly-head intensity is grows slightly with increasing $F_{0}$ and decreasing $\gamma$ for all the time after the injection. The maximum variations occur in the first second, and then they gradually decay. The Ba (Figure II) and Pa continuum intensity distributions are greatly affected by the non-thermal impacts. In the first second they engender strong rising of the $\mathrm{Ba}$ and $\mathrm{Pa}$-head intensities, particularly, for high $F_{0}$ and small $\gamma$. The head intensity slopes are also increased by the non-thermal effects. It is interesting that for the beams with initial fluxes $F_{0}=10^{11} \mathrm{erg} \mathrm{cm} \mathrm{cm}^{-2} \mathrm{~s}^{-1}$ and $\gamma=3$ we see almost a straight line in Figure II in spite of the varying slope over wavelength for other parameters. Later $(t>4 \mathrm{~s})$ the effect in the $\mathrm{Ba}$ and $\mathrm{Pa}$ continuum intensities is smaller because of the increasing role of the thermal processes due to the gas-dynamic processes.

\section{CONCLUSIONS}

The non-thermal effects in the hydrogen continua give an opportunity to study behaviour of electron beams in deep layers to which they penetrate into the chromosphere. The non-thermal processes are probablely responsible for a temporal correlation between bursts observed in the hard X-rays and in UV-spectra during the beam injections in the impulsive solar flares. The events of smaller scales on the sun (micro- and nanoflares) and of greater scales on flare stars are candidates for the coronal heating mechanism and solar and stellar wind origin. If the solar flares and these flare-like phenomena have the same non-thermal origin we can achieve a better understanding of the fundamental problems on both the sun and the stars.

The authors are very grateful to the LOC of the IAU Colloquium 137 and to Prof. W.Weiss for financial support and the opportunity to take a part in the meeting.

\section{REFERENCES}

Aboudarham,J. \& Henoux,J.-C.: 1986, Astron.Astrophys. 156, 73

Somov,B.V., Spektor,A.R. \& Syrovatskii, S.I.: 1981, Solar Phys. 73, 145

Zharkova,V.V.\& Kobylinsky,V.A.: 1989, Soviet Astron.Lett. 15, 366

Zharkova,V.V. \& Kobylinsky,V.A.: 1991, Soviet Astron.Lett. 17, 34

Zharkova,V.V. \& Kobylinsky,V.A.: 1992, Kinemat. and phys. of space bodies 8 No 3 (in Russian)

Zharkova,V.V. \& Kobylinsky,V.A.: 1993, Solar Phys. (submitted) 